(c) Elsevier/INRA

Original article

\title{
Estimating genetic parameters using an animal model with imaginary effects
}

\author{
R Thompson ${ }^{1 *}, \mathrm{~K}$ Meyer $^{2}$ \\ 1 AFRC Institute of Animal Physiology and Genetics, Edinburgh Research Station, \\ Roslin, Midlothian, EH25 9PS; \\ 2 Institute of Animal Genetics, University of Edinburgh, King's Buildings, \\ Edinburgh EHg $3 J N, U K$
}

(Received 8 July 1988; accepted 24 November 1989)

\begin{abstract}
Summary - The estimation of additive genetic variance by maximum likelihood is discussed. The extension of reduced animal models, when parents are not inbred to allow the use of existing algorithms for maximum likelihood estimation of additive genetic variance, is described. This involves the introduction of imaginary effects with negative variance, and leads to computation using complex arithmetic. Methods are developed to allow the computation to be carried out using only real arithmetic. This method has computational advantages when only a small proportion of animals have offspring.
\end{abstract}

genetic parameter / animal model / estimation / maximum likelihood

Résumé - Estimation de paramètres génétiques selon un modẻle animal incluant des effets imaginaires - On discute l'estimation de la variance génétique additive par le maximum de vraisemblance. On décrit l'extension des algorithmes disponibles d'estimation par le maximum de vraisemblance de la variance génétique additive, à la situation d'un modèle animal réduit au cas où les parents ne sont pas consanguins. Ceci passe par l'introduction d'effets imaginaires, de variance négative, et conduit à des calculs en arithmétique complexe. Des méthodes sont développées pour n'utiliser que l'arithmétique réelle. Cette méthode présente des avantages numériques quand une faible proportion seulement des animaux ont des descendants.

paramètre génétique / modèle animal / estimation / maximum de vraisemblance

\section{INTRODUCTION}

Additive genetic variance and heritability have most commonly been estimated in animal breeding data information from collateral relatives, such as half-sibs or fullsibs, or non-collateral relatives, such as parent-offspring. Covariances generated by these relationships provide the most information for estimating additive genetic variance. However, there is interest in combining these alternative estimates

* Correspondence and reprints 
(Nicholas and Hill, 1974), and also using the additional information from other genetic relationships.

Linear models can be formed that contain genetic and environmental effects for each animal, commonly called individual animal models (eg, Quaas and Pollak, 1980). In theory at least, standard or restricted maximum likelihood estimation (ML or REML) procedures can be applied to individual animal models for variance component estimation (Harville, 1977). One problem is that this model generates as many equations as there are animals in the pedigree and so there have been attempts to reduce the computational effort. Thompson (1977) considered the case of two generations and showed how to develop REML estimating equations just for animals in the first generation. More generally, this estimation can usually be interpreted using predicted breeding values. Quaas and Pollak (1980) showed that a reduced animal model (RAM) has advantages in calculating predicted breeding values. Notably, only equations for animals with offspring are needed. Henderson (1986) and Sorensen and Kennedy (1986) showed that REML and minimum norm quadratic (MINQUE) estimation can be expressed with advantage using a RAM.

However, these (REML) methods are iterative and, as presented, require the inversion of a matrix with as many rows and columns as the number of parents in each round of iteration. For some variance component problems, this computational burden can be reduced by using orthogonal transformations to give simpler equations, either in terms of a diagonal matrix (Patterson and Thompson, 1971; Dempster et al, 1984), or in terms of a tridiagonal matrix (Smith and Graser, 1986). These results are not directly applicable to estimation methods based on a RAM. This paper shows how a RAM can be modified by the introduction of extra random effects with negative variances, so that the resulting matrices involved in estimation can be tridiagonalized using existing algorithms based on Householder transformations. Complex numbers are needed to take account of negative variances, but a new algorithm is given that takes account of the special structure of the matrices involved, and reduces the need for arithmetic based on complex numbers.

\section{THE MODEL}

If additive genetic covariances are the only source of covariances between records, then a linear model for the individual animal model (IAM) can be written as:

$$
\mathbf{y}=\mathbf{X b}+\mathbf{Z a}+\mathbf{e}
$$

with $E(\mathbf{y})=\mathbf{X b}, E(\mathbf{a})=E(\mathbf{e})=0$ and $V(\mathbf{a})=\mathbf{A} \sigma_{A}^{2}, V(\mathbf{e})=\mathbf{I} \sigma_{E}^{2}$ and $\operatorname{cov}\left(\mathbf{a}, \mathbf{e}^{\prime}\right)=0$, where $\mathbf{y}, \mathbf{b}, \mathbf{a}$ and $\mathbf{e}$ denote the vectors of observations, fixed effects, animal effects and residual errors. $\mathbf{X}$ and $\mathbf{Z}$ are the corresponding incidence matrices, and for simplicity, we assume $\mathbf{X}$ had $r$ columns and rank $r$. With single records per animal, $\mathbf{Z}=\mathbf{I}_{m}$, where $m$ denotes the number of observations. $\mathbf{A}$ is the numerator relationship matrix (NRM) between animals.

As described by Quaas and Pollak (1980), with a RAM, the vector of animals is divided into parents, $i e$, animals which have offspring (subscript $P$ in the following), and non-parents, $i e$, animals without progeny (denoted by subscript $N$ ). The additive genetic value for non-parents is then partitioned into contributions from parents and parts due to the Mendelian sampling. Including the latter, together 
with the residual $\mathbf{e}$ in eqn(1) into a new residual error, gives the RAM as a reparameterisation of eqn(1):

$$
\left[\begin{array}{l}
\mathbf{y}_{P} \\
\mathbf{y}_{N}
\end{array}\right]=\left[\begin{array}{l}
\mathbf{X}_{P} \\
\mathbf{X}_{N}
\end{array}\right] \mathbf{b}+\left[\begin{array}{l}
\mathbf{I}_{P} \\
\mathbf{z}_{N}
\end{array}\right] \mathbf{a}_{P}+\left[\begin{array}{l}
\mathbf{e}_{P} \\
\mathbf{e}_{N}
\end{array}\right]
$$

Let $p$ denote the number of parents and $q$ the number of non-parents, then $(p+q=m) . \mathbf{Z}_{N}$ is then a matrix of order $q x p$, with elements $z_{i j}=0.5$, if $j$ is a parent of $i$, and zero otherwise. This representation, strictly speaking, assumes all parents of non-parents are in the data and one record per individual. However, eqn(2) can be easily modified, if that is not the case. The residual error for a non-parent has variance $\sigma_{W}^{2}=\sigma_{E}^{2}+0.5 \sigma_{A}^{2}$, if both parents are known, and variance $\sigma_{W}^{2}=\sigma_{E}^{2}+0.75 \sigma_{A}^{2}$, if only one parent is known, provided they are not inbred. In the following, it is assumed that there is equal parental information for all non-parents and that parents are non inbred $i e$, that $\sigma_{W}^{2}$ is constant.

Estimation equations, based on the RAM, usually have to be reconstructed after each variance iteration because the ratio of error to additive variance changes. Therefore, for computational reasons, it is desirable to have a model where the vector of residuals has homogeneous variance. This can be achieved by a further reparameterisation, adding effects to either parents or non-parents. As there are usually fewer parents than non-parents, fewer equations will be generated if eqn(2) is expanded to:

$$
\left[\begin{array}{l}
\mathbf{y}_{P} \\
\mathbf{y}_{N}
\end{array}\right]=\left[\begin{array}{l}
\mathbf{X}_{P} \\
\mathbf{X}_{N}
\end{array}\right] b+\left[\begin{array}{l}
\mathbf{I}_{P} \\
\mathbf{Z}_{N}
\end{array}\right] a_{P}+\left[\begin{array}{c}
\mathbf{e}_{I} \\
0
\end{array}\right]+\left[\begin{array}{c}
\mathbf{e}_{P}-\mathbf{e}_{I} \\
\mathbf{e}_{N}
\end{array}\right]
$$

The variance of $\mathbf{e}_{I}$ is such that $\operatorname{var}\left(\mathbf{e}_{I}\right)+\operatorname{var}\left(\mathbf{e}_{P}-\mathbf{e}_{I}\right)=\operatorname{var}\left(\mathbf{e}_{P}\right)=\mathbf{I}_{P} \sigma_{E}^{2}$ and $\operatorname{var}\left(\mathbf{e}_{P}-\mathbf{e}_{I}\right)=\mathbf{I} \sigma_{w}^{2}$, so that $\mathbf{e}_{P}-\mathbf{e}_{I}$ and $\mathbf{e}_{N}$ have the same variance. Hence, $\operatorname{var}\left(\mathbf{e}_{I}\right)=-c^{2} \sigma_{A}^{2} \mathbf{I}_{P}$, with $c^{2}=0.75$ and 0.50 for one and both parents known, respectively. Because of the assumption that there is equal parental information for all non-parents, $c^{2}$ is the same for all $\mathbf{e}_{I}$ values. Normally, variances are assumed to be positive so there is a slight difficulty in interpreting $\mathbf{e}_{I}$ with a negative variance. However, if $\mathbf{a}_{D}$ are effects with variance $\sigma_{A}^{2} I_{P}$, then defining $\mathbf{e}_{I}=i c \mathbf{a}_{D}$, where $i=\sqrt{-1}$, then the variance of $\mathbf{e}_{I}=-c^{2} \sigma_{A}^{2} I_{P}$. Hence, $\mathbf{e}_{I}$ can be thought of as imaginary effects as they are a multiple of $i$.

Hence, eqn(3) can be written in terms of real effects as:

$$
\left[\begin{array}{l}
\mathbf{y}_{P} \\
\mathbf{y}_{N}
\end{array}\right]=\left[\begin{array}{l}
\mathbf{X}_{P} \\
\mathbf{X}_{N}
\end{array}\right] b+\left[\begin{array}{l}
\mathbf{I}_{P} \\
\mathbf{Z}_{N}
\end{array}\right] a_{P}+\left[\begin{array}{c}
i c \mathbf{I}_{P} \\
0
\end{array}\right] \mathbf{a}_{D}+\mathbf{e}
$$

with $\mathbf{e}=\left[\left(\mathbf{e}_{P}^{\prime}-\mathbf{e}_{I}^{\prime}\right) \mathbf{e}_{N}^{\prime}\right]^{\prime}$.

This gives mixed model equations (MME) as:

$$
\left[\begin{array}{ccc}
\mathbf{X}_{P}^{\prime} \mathbf{X}_{P}+\mathbf{X}_{N}^{\prime} \mathbf{X}_{N} & \mathbf{X}_{P}^{\prime}+\mathbf{X}_{N}^{\prime} \mathbf{Z}_{N} & i c \mathbf{X}_{P}^{\prime} \\
\mathbf{X}_{P}+\mathbf{Z}_{N}^{\prime} \mathbf{X}_{N} & \mathbf{I}_{P}+\mathbf{Z}_{N}^{\prime} \mathbf{Z}_{N}+\lambda \mathbf{A}^{-1} & i c \mathbf{I}_{P} \\
i c \mathbf{X}_{P} & i c \mathbf{I}_{P} & -c^{2} \mathbf{I}_{P}+\lambda \mathbf{I}_{P}
\end{array}\right]\left[\begin{array}{c}
\mathbf{b} \\
\mathbf{a}_{P} \\
\mathbf{a}_{D}
\end{array}\right]=\left[\begin{array}{c}
\mathbf{X}_{P}^{\prime} \mathbf{y}_{P}+\mathbf{X}_{N}^{\prime} \mathbf{y}_{N} \\
\mathbf{y}_{P}+\mathbf{Z}_{N}^{\prime} \mathbf{y}_{N} \\
i c \mathbf{y}_{P}
\end{array}\right]
$$

where $\lambda$ denotes the variance ratio $\sigma_{W}^{2} / \sigma_{A}^{2}$, and $\mathbf{A}_{P}$ is the numerator relationship matrix of parents. 
Eliminating $b$ from eqn(4) gives equations of the form

$$
\left[\begin{array}{cc}
\mathbf{B}+\lambda \mathbf{A}_{P}^{-1} & i \mathbf{C} \\
i \mathbf{C}^{\prime} & \mathbf{D}+\lambda \mathbf{I}_{P}
\end{array}\right]\left[\begin{array}{c}
\mathbf{a}_{P} \\
\mathbf{a}_{D}
\end{array}\right]=\left[\begin{array}{c}
\mathbf{f} \\
i \mathbf{g}
\end{array}\right]
$$

where

$$
\begin{aligned}
& \mathbf{B}=\mathbf{I}_{P}+\mathbf{Z}_{N}^{\prime} \mathbf{Z}_{N}-\left(\mathbf{X}_{P}+\mathbf{Z}_{N}^{\prime} \mathbf{X}_{N}\right)\left(\mathbf{X}_{P}^{\prime} \mathbf{X}_{P}+\mathbf{X}_{N}^{\prime} \mathbf{X}_{N}\right)^{-1}\left(\mathbf{X}_{P}^{\prime}+\mathbf{X}_{N}^{\prime} \mathbf{Z}_{N}\right) \\
& \mathbf{C}=c \mathbf{I}_{P}-c\left(\mathbf{X}_{P}+\mathbf{Z}_{N}^{\prime} \mathbf{X}_{N}\right)\left(\mathbf{X}_{P}^{\prime} \mathbf{X}_{P}+\mathbf{X}_{N}^{\prime} \mathbf{X}_{N}\right)^{-1} \mathbf{X}_{P}^{\prime} \\
& \mathbf{D}=-c^{2} \mathbf{I}_{P}+c^{2} \mathbf{X}_{P}\left(\mathbf{X}_{P}^{\prime} \mathbf{X}_{P}+\mathbf{X}_{N}^{\prime} \mathbf{X}_{N}\right)^{-1} \mathbf{X}_{P}^{\prime} \\
& \mathbf{f}=\mathbf{y}_{P}+\mathbf{Z}_{N}^{\prime} \mathbf{y}_{N}-\left(\mathbf{X}_{P}+\mathbf{Z}_{N}^{\prime} \mathbf{X}_{N}\right)\left(\mathbf{X}_{P}^{\prime} \mathbf{X}_{P}+\mathbf{X}_{N}^{\prime} \mathbf{X}_{N}\right)^{-1}\left(\mathbf{X}_{P}^{\prime} \mathbf{y}_{P}+\mathbf{X}_{N}^{\prime} \mathbf{y}_{N}\right) \\
& \mathbf{g}=c \mathbf{y}_{P}-c \mathbf{X}_{P}\left(\mathbf{X}_{P}^{\prime} \mathbf{X}_{P}+\mathbf{X}_{N}^{\prime} \mathbf{X}_{N}\right)^{-1}\left(\mathbf{X}_{P}^{\prime} \mathbf{y}_{P}+\mathbf{X}_{N}^{\prime} \mathbf{y}_{N}\right)
\end{aligned}
$$

Hence,

$$
\left[\begin{array}{l}
\mathbf{a}_{P} \\
\mathbf{a}_{D}
\end{array}\right]=\left[\begin{array}{cc}
\mathbf{B}+\mathbf{A}_{P}^{-1} & i \mathbf{C} \\
i \mathbf{C}^{\prime} & \mathbf{D}+\lambda \mathbf{I}_{P}
\end{array}\right]^{-1}\left[\begin{array}{c}
\mathbf{f} \\
i \mathbf{g}
\end{array}\right]=\left[\begin{array}{cc}
\mathbf{H}_{11} & i \mathbf{H}_{12} \\
i \mathbf{H}_{12}^{\prime} & \mathbf{H}_{22}
\end{array}\right]\left[\begin{array}{c}
\mathbf{f} \\
i \mathbf{g}
\end{array}\right]
$$

The component matrices $\mathbf{H}_{11}, \mathbf{H}_{12}$ and $\mathbf{H}_{22}$ are all real.

Equation (7) gives predictors of the effects $\mathbf{a}_{P}$ and $\mathbf{a}_{D}$. Note that $\mathbf{a}_{P}$ is real and $\mathbf{a}_{D}$ is imaginary, so that $i \mathbf{a}_{R}=\mathbf{a}_{D}$ with $\mathbf{a}_{R}$ real, and the predictor for $\mathbf{e}_{I}=-c \mathbf{a}_{R}$ is a real quantity, as would be anticipated. REML equations to estimate $\sigma_{A}^{2}$ and $\sigma_{W}^{2}$ are (in terms of real quantities):

$$
\begin{aligned}
& \sigma_{A}^{2}=\left[\mathbf{a}_{P}^{\prime} \mathbf{A}_{P}^{-1} \mathbf{a}_{P}-\mathbf{a}_{R}^{\prime} \mathbf{a}_{R}\right] /\left[n-\operatorname{tr}\left(\mathbf{A}_{P}^{-1} C_{11}\right)-\operatorname{tr}\left(C_{22}\right)\right] \\
& \mathbf{R}=\mathbf{y}_{P}^{\prime} \mathbf{y}_{P}+\mathbf{y}_{N}^{\prime} \mathbf{y}_{N}-\left(\mathbf{y}_{P}^{\prime} \mathbf{X}_{P}+\mathbf{y}_{N}^{\prime} \mathbf{X}_{N}\right)\left(\mathbf{X}_{P}^{\prime} \mathbf{X}_{P}+\mathbf{X}_{N}^{\prime} \mathbf{X}_{N}\right)^{-1}\left(\mathbf{X}_{P}^{\prime} \mathbf{y}_{P}+\mathbf{X}_{N}^{\prime} \mathbf{y}_{N}\right)
\end{aligned}
$$

$$
\begin{aligned}
\sigma_{W}^{2}=\left[\mathbf{R}-\mathbf{a}_{P}^{\prime} \mathbf{f}+\mathbf{a}_{R}^{\prime} \mathbf{g}-\right. & \left.\mathbf{a}_{P}^{\prime} \mathbf{A}_{P}^{-1} \mathbf{a}_{P}+\mathbf{a}_{R}^{\prime} \mathbf{a}_{R}\right] /[m-r-n \\
& \left.+\operatorname{tr}\left(\mathbf{A}_{P}^{-1} \mathbf{H}_{11}\right)+\operatorname{tr}\left(\mathbf{H}_{22}\right)\right]
\end{aligned}
$$

where $r$ is the rank of $\left(\mathbf{X}_{P}^{\prime} \mathbf{X}_{P}+\mathbf{X}_{N}^{\prime} \mathbf{X}_{N}\right)$, and $n$ is the number of elements in both $\mathbf{a}_{P}$ and $\mathbf{a}_{D}$.

These equations involve $\sigma_{A}^{2}$ and $\sigma_{W}^{2}$ through $\lambda=\sigma_{W}^{2} / \sigma_{A}^{2}$ on both sides of eqns(8) and (10), and have to be solved iteratively. To simplify eqns(8) and (10) and avoid the inversion associated with $A_{P}^{-1}$, Smith and Graser (1986) and Meyer (1987) suggest writing $\mathbf{A}_{P}$ as $\mathbf{L} \mathbf{L}^{\prime}$ and using $\mathbf{a}_{L}=\mathbf{L}^{-1} \mathbf{a}_{P}$ in a model equivalent to eqn(3). The effects $\mathbf{a}_{L}$ are uncorrelated. Using Meyer's results and eqns(8) and (10), gives an alternative form of the EM algorithm.

$$
\begin{aligned}
& \sigma_{A}^{2}=\left[\mathbf{a}_{L}^{\prime} \mathbf{a}_{L}-\mathbf{a}_{R}^{\prime} \mathbf{a}_{R}\right] /\left[n-\operatorname{tr}\left(\mathbf{H}_{11}^{*}\right)-\operatorname{tr}\left(\mathbf{H}_{22}^{*}\right)\right] \\
& \sigma_{W}^{2}=\left[R-\mathbf{a}_{L}^{\prime} \mathbf{L}^{\prime} \mathbf{f}+\mathbf{a}_{R}^{\prime} \mathbf{g}-\mathbf{a}_{L}^{\prime} \mathbf{a}_{L}+\mathbf{a}_{R}^{\prime} \mathbf{a}_{R}\right] /\left[m-r-n+\operatorname{tr}\left(\mathbf{H}_{11}^{*}\right)+\operatorname{tr}\left(\mathbf{H}_{22}^{*}\right)\right]
\end{aligned}
$$


where $\left[\begin{array}{c}\mathbf{a}_{L} \\ \mathbf{a}_{D}\end{array}\right]=\left[\begin{array}{cc}\mathbf{L}^{\prime} \mathbf{B L}+\lambda \mathbf{I} & i \mathbf{L}^{\prime} \mathbf{C} \\ i \mathbf{C}^{\prime} \mathbf{L} & \mathbf{D}+\lambda \mathbf{I}_{P}\end{array}\right]^{-1}\left[\begin{array}{c}\mathbf{L}^{\prime} f \\ i \mathbf{g}\end{array}\right]=\left(\mathbf{A}_{1}+\mathbf{I} \lambda\right)^{-1}\left[\begin{array}{c}\mathbf{L}^{\prime} f \\ i \mathbf{g}\end{array}\right]$

$$
=\left[\begin{array}{cc}
\mathbf{H}_{11}^{*} & i \mathbf{H}_{12}^{*} \\
i \mathbf{H}_{12}^{\prime *} & \mathbf{H}_{22}^{*}
\end{array}\right]\left[\begin{array}{c}
\mathbf{L}^{\prime} f \\
i \mathbf{g}
\end{array}\right]
$$

where $\mathbf{A}_{1}=\left[\begin{array}{cc}\mathbf{L}^{\prime} \mathbf{B L} & i \mathbf{L}^{\prime} \mathbf{C} \\ i \mathbf{C}^{\prime} \mathbf{L} & \mathbf{D}\end{array}\right]$

( $\mathbf{A}_{1}$ is not to be confused with $\mathbf{A}$ or $\mathbf{A}_{p}$, numerator relationship matrices).

This iterative scheme requires the inversion of $\left(\mathbf{A}_{1}+\mathbf{I} \lambda\right)$ in each iteration. Smith and Graser (1986) have shown that these computations can be reduced by writing $\mathbf{A}_{1}$ as $\left(\mathbf{P A}_{n}^{*} \mathbf{P}^{\prime}\right)$ where $\mathbf{P}$ is an orthogonal matrix and $\mathbf{A}_{n}^{*}$ is a tridiagonal matrix. Using algebra similar to that of Smith and Graser (1986), it can be shown that quantities arising in eqns(11) and (12) can be written in terms of $\left(\mathbf{A}_{n}^{*}+\lambda \mathbf{I}\right)^{-1}$ and $\mathbf{q}^{*}=\mathbf{P}^{\prime}\left[\begin{array}{c}\mathbf{L}^{\prime} \mathbf{f} \\ i \mathbf{g}\end{array}\right]$. For example:

$$
\begin{aligned}
& \mathbf{a}_{L}^{\prime} \mathbf{a}_{L}-\mathbf{a}_{R}^{\prime} \mathbf{a}_{R}=\mathbf{a}_{M}^{\prime} \mathbf{a}_{M} \\
& \mathbf{a}_{L}^{\prime} \mathbf{L} \mathbf{f}^{\prime}-\mathbf{a}_{R}^{\prime} \mathbf{g}=\mathbf{a}_{M}^{\prime} \mathbf{q}^{*}
\end{aligned}
$$

and $\operatorname{tr}\left(\mathbf{H}_{11}^{*}\right)+\operatorname{tr}\left(\mathbf{H}_{22}^{*}\right)=\operatorname{tr}\left[\left(\mathbf{A}_{n}^{*}+\lambda \mathbf{I}\right)^{-1}\right]$

$\mathbf{A}_{n}^{*}$ can be found using a sequence of Householder transformations, using complex arithmetic. In the Appendix, it is shown how the special structure of $\mathbf{A}_{1}$ with real quadrants $\left(\mathbf{L}^{\prime} \mathbf{B L}\right.$ and $\left.\mathbf{D}\right)$ and imaginary quadrants $\left(i \mathbf{L}^{\prime} \mathbf{C}\right)$ and $\left(i \mathbf{L}^{\prime} \mathbf{C}\right)^{\prime}$ can be used to give an algorithm for $\mathbf{A}_{n}^{*}$ and $\mathbf{q}^{*}$ and to evaluate eqns(12) to (16), using only real arithmetic. It should be noted that this computational strategy is using an existing iterative scheme and is manipulating the equations to reduce the number of computations.

\section{NUMERICAL EXAMPLE}

To illustrate the formation of the model and mixed model equations, we use an example of 5 observations of the same sex with individuals 2 and 3 , the offspring of individual 1 , and individuals 4 and 5 , the offspring of individual 2 . There is 1 fixed effect with 2 levels, the first 3 observations at 1 level, and the last 2 at the second level. The model (4) is then of the form:

$$
\left[\begin{array}{l}
y_{1} \\
y_{2} \\
y_{3} \\
y_{4} \\
y_{5}
\end{array}\right]=\left[\begin{array}{ll}
1 & 0 \\
1 & 0 \\
1 & 0 \\
0 & 1 \\
0 & 1
\end{array}\right]\left[\begin{array}{l}
b_{1} \\
b_{2}
\end{array}\right]+\left[\begin{array}{cc}
1 & 0 \\
0 & 1 \\
1 / 2 & 0 \\
0 & 1 / 2 \\
0 & 1 / 2
\end{array}\right]\left[\begin{array}{l}
a_{P 1} \\
a_{P 2}
\end{array}\right]+i 0.866\left[\begin{array}{ll}
1 & 0 \\
0 & 1 \\
0 & 0 \\
0 & 0 \\
0 & 0
\end{array}\right]\left[\begin{array}{l}
a_{D 1} \\
a_{D 2}
\end{array}\right]+\left[\begin{array}{l}
e_{1} \\
e_{2} \\
e_{3} \\
e_{4} \\
e_{5}
\end{array}\right]
$$


with the observation vector coded so that observations on parents ( 1 and 2) occur first. The variance of $\mathbf{e}_{i}$ is $\sigma_{E}^{2}+3 / 4 \sigma_{A}^{2}$, as animals have only one known parent. The variance of $\left(y_{1}\right)=\sigma_{A}^{2}-3 / 4 \sigma_{A}^{2}+\sigma_{E}^{2}+3 / 4 \sigma_{A}^{2}=\sigma_{E}^{2}+\sigma_{A}^{2}, \operatorname{var}\left(y_{5}\right)=$ $1 / 4 \sigma_{A}^{2}+\sigma_{E}^{2}+3 / 4 \sigma_{A}^{2}=\sigma_{E}^{2}+\sigma_{A}^{2}$.

$$
\text { If } \lambda=3 \text {, then } \lambda \mathbf{A}_{P}^{-1}=\left[\begin{array}{rr}
4 & -2 \\
-2 & 4
\end{array}\right] \text { and eqn(5) becomes: }
$$

$$
\left[\begin{array}{rrrrrr}
3 & 0 & 1.5 & 1 & 0.866 i & 0.866 i \\
0 & 2 & 0 & 1 & 0 & 0 \\
1.5 & 0 & 5.25 & -2 & 0.866 i & 0 \\
1 & 1 & -2 & 5.5 & 0 & 0.866 i \\
0.866 i & 0 & 0.866 i & 0 & 2.25 & 0 \\
0.866 i & 0 & 0 & 0.866 i & 0 & 2.25
\end{array}\right]\left[\begin{array}{c}
b_{1} \\
b_{2} \\
a_{P 1} \\
a_{P 2} \\
a_{D 1} \\
a_{D 2}
\end{array}\right]=\left[\begin{array}{l}
y_{1}+y_{2}+y_{3} \\
y_{4}+y_{5} \\
y_{1}+0.5 y_{3} \\
y_{2}+0.5\left(y_{4}+y_{5}\right) \\
0.866 i y_{1} \\
0.866 i y_{2}
\end{array}\right]
$$

Eliminating $b_{1}$ and $b_{2}$ gives:

$$
\left[\begin{array}{rrrr}
4.500 & -2.500 & 0.433 i & -0.433 i \\
-2.500 & 4.666 & -0.289 i & 0.578 i \\
0.433 i & -0.289 i & 2.500 & 0.250 \\
-0.433 i & 0.578 i & 0.250 & 2.500
\end{array}\right]\left[\begin{array}{l}
a_{P 1} \\
a_{P 2} \\
a_{D 1} \\
a_{D 2}
\end{array}\right]=\left[\begin{array}{l}
\left(y_{1}-y_{2}\right) / 2 \\
\left(-y_{1}+2 y_{2}-y_{3}\right) / 3 \\
0.866 i\left(2 y_{1}-y_{2}-y_{3}\right) / 3 \\
0.866 i\left(-y_{1}+2 y_{2}-y_{3}\right) / 3
\end{array}\right]
$$

This shows the structure of eqn(6) with two real quadrants and two imaginary quadrants in the matrix on the left hand side. For this example, estimates of the effects can be found by partitioning the coefficient matrix into $2 \times 2$ real and imaginary parts and using results on inverses of partitioned matrices.

\section{DISCUSSION}

This is a novel approach with the advantage of working with matrices of size $2 p$, rather than $(n+p)$, and the computations involved, are of the order of $(2 p)^{3}$, rather than $(n+p)^{3}$. This technique is, therefore, of more use in populations with a low proportion of animals used as parents. The technique can be easily extended to estimates 2 multivariate residual and additive variance components matrices when measurements are taken on all animals using the procedures developed by Meyer (1985).

Two assumptions are made. First, that all non-parents have equal parental information. If this assumption is not satisfied, non-parents with unknown parents can have dummy parents inserted into the model. In most animal breedings sets where inbreeding is consciously avoided, the second assumption (parents are not inbred) is unlikely to be important. There is more likely to be concern about residual variance homogeneity, than about inbreeding generated genetic variance homogeneity.

Graser et al (1987), suggested a derivative-free method of estimating variance components, based on sequentially calculating the likelihood. Their method is an 
obvious competitor, but it is difficult to say precisely when each method is to be preferred. The time required, depends on the number of animals, structure of population the sequence used in calculating the likelihood, the number of variates measured and the speed of convergence of the iterative procedure. Their comments suggest that our method could be advantageous if the number of parents is less than 350 .

The method has been presented for a model with additive genetic covariances, but in many cases, other components, such as litter variances, need estimation. In such cases, the procedure can be used for a given ratio of litter variance to residual variance and repeated for different values of the ratio, in a similar manner to that suggested by Smith and Graser (1986).

\section{REFERENCES}

Dempster AP, Selwyn MR, Patel IM, Roth AJ (1984) Statistical and computational aspects of mixed model analysis. Appl Stat 33, 203-214

Graser H-U, Smith SP, Tier B (1987) A derivative-free approach for estimating variance components in animal models by restricted maximum likelihood. J Anim Sci 64, 1362-1370

Harville DA (1977) Maximum likelihood approaches to variance component estimation and to related problems. J Am Stat Assoc 72, 320-338

Henderson CR (1986) Estimation of variances in Animal Model and Reduced Animal Model for single traits and single records. J Dairy Sci 60, 1394-1402

Meyer K (1985) Maximum likelihood estimation of variances components for a multivariate mixed model with equal design matrices. Biometrics 41, 153-166

Meyer K (1987) A note on the use of an equivalent model to account for relationships between animals in estimating variance components. J Anim Breed Genet 104, 163168

Nicholas FW, Hill WG (1974) Estimation of heritability by both regression of offspring on parent and intra-class correlation of sibs in one experiment. Biometrics 30, 447-68

Patterson HD, Thompson R (1971) Recovery of inter-block information when block sizes are unequal. Biometrika 58, 545-554

Quaas RL, Pollack EJ (1980) Mixed model methodology for farm and ranch beef cattle testing programs. J Anim Sci 51, 1277-1287

Smith SP, Graser H-U (1986) Estimating variance components in a class of mixed models by restricted maximum likelihood. $J$ Dairy Sci $69,1156-1165$

Sorensen DA, Kennedy BW (1986) Analysis of selection experiments using mixed model methodology. J Anim Sci 63, 245-258

Thompson R (1977) The estimation of heritability with unbalanced data. II. Data available on more than two generations. Biometrics, 33, 497-504

Wilkinson JH, Reinsch C (1971) Linear Algebra. Springer-Verlag, Berlin 


\section{APPENDIX}

\section{Reduction of complex matrices to tridiagonal form}

A square matrix is said to be tridiagonal (in the first $t$ rows) if the only non-zero elements are in the $r, s$ elements, where $s=r-1, r$, or $r+1(r<t)$. A real symmetric matrix $\mathbf{A}_{1}$, of size $n \times n$, can be reduced to tridiagonal form $\mathbf{A}_{n-1}$ by a sequence of $(n-2)$ Householder transformations (for example, Wilkinson and Reinsch, 1971).

In this sequence of Householder transformations, at the $t^{\text {th }}$ stage, the transformation $\mathbf{P}_{t}$ is chosen to make the $t^{\text {th }}$ row of $\mathbf{A}_{t+1}$ contain all zero elements, except possibly those in the $(t-1), t$, and $(t+1)$ columns. This operation will be called pivoting on the $t^{\text {th }}$ row. The non-zero elements in the $j^{\text {th }}(1<j<n)$ row of $\mathbf{A}_{n-1}$ are in the $(j-1)^{\text {th }}$ and $(j+1)^{\text {th }}$ columns, indicating the previous $(j-1)$ and next $(j+1)$ pivots.

For the algorithm presented in this paper, a complex matrix $\mathbf{A}_{1}$ of the form:

$$
\mathbf{A}_{t}=\left[\begin{array}{cc}
\mathbf{B} & i \mathbf{C} \\
i \mathbf{C}^{\prime} & \mathbf{D}
\end{array}\right]
$$

where $\mathbf{B}, \mathbf{C}, \mathbf{D}$ are real matrices [of size $\left(n_{1} \times n_{1}\right),\left(n_{1} \times n_{2}\right)$ and $\left(n_{2} \times n_{2}\right)$ ] is to be tridiagonalized.

In order to illustrate the method, a numerical example will be given.

The matrix $\mathbf{A}_{1}$ satisfying eqn(A1) with:

$$
\begin{aligned}
B & =\left[\begin{array}{rrrr}
3.00 & & & \\
-1.50 & 3.00 & & \\
-0.75 & -0.75 & 3.00 & \\
-0.75 & -0.75 & -1.50 & 3.00
\end{array}\right] \\
C^{\prime} & =\left[\begin{array}{rrrr}
1.50 & -0.50 & -0.50 & -0.50 \\
-0.50 & 1.50 & -0.50 & -0.50 \\
-0.50 & -0.50 & 1.50 & -0.50 \\
-0.50 & -0.50 & -0.50 & 1.50
\end{array}\right] \\
D & =\left[\begin{array}{rrrr}
-1.20 & & & \\
0.30 & -1.20 & & \\
0.30 & 0.30 & -1.20 & \\
0.30 & 0.30 & 0.30 & -1.20
\end{array}\right]
\end{aligned}
$$

will be used, with $n_{1}=n_{2}=4$ (for convenience, when matrices are symmetrical, only the lower triangular part is given). The sequence of Householder transformations derived for real matrices could be used but, as a square root of a sum of squares, $\sigma_{t}$, is used and as this could be negative, this would involved complex arithmetic, which is computationally considerably more demanding than real arithmetic. A modification of the tridiagonalization is now presented which avoids complex calculations. 
There are two stages involved. In the first, a sequence of Householder transformation is used that gives $\mathbf{A}_{t+1}=\mathbf{P}_{t} \mathbf{A}_{t} \mathbf{P}_{t}^{\prime}$, where $\mathbf{A}_{t+1}$ and $\mathbf{P}_{t}$ are found to have the same form as $\mathbf{A}$ in eqn(A1) with quadrants of real and imaginary numbers. This involves changing the ordering of the pivoting. The rows of $\mathbf{A}_{1}$ are split into two sets 1 to $n_{1}$ and $\left(1+n_{1}\right)$ to $\left(n_{1}+n_{2}\right)$, corresponding to the division into quadrants in eqn(A1). The pivoting is started on row 1 and continues in the first set until $\sigma_{t}<0$, then we pivot on the first row of the second set and continue in this set until again $\sigma_{t}<0$. The process is then repeated until $n_{1}+n_{2}-1=n-1$ rows have been pivoted on. This procedure produces a matrix $\mathbf{A}_{t+1}$ with as many nonzero elements as the usual procedure, but $\mathbf{A}_{t+1}$ is not necessarily tridiagonal. For example, for the numerical example, the ordering of pivoting is found to be rows 1 , $2,5,3,6,7$, giving $\mathbf{A}_{7}$.

$\left[\begin{array}{lllllrrrr}3.0000 & & & & & & & \\ 0.6124 & 9.5000 & & & & & & \\ 0.0000 & 0.0000 & 0.7969 & & & & & \\ 0.0000 & 0.0000 & 0.0000 & 3.7419 & & & & \\ 0.0000 i & 8.7106 i & 0.3864 i & 0.0000 i & -8.3081 & & & \\ 0.0000 i & 0.0000 i & 0.2230 i & 0.0000 i & 0.0000 & -0.4888 & & \\ 0.0000 i & 0.0000 i & 0.0000 i & 0.1518 i & 0.0000 & 0.0000 & -0.5229 & \\ 0.0000 i & 0.0000 i & 0.0000 i & 0.0000 i & 0.0000 & 0.0000 & 0.2179 & -0.5190\end{array}\right]$

There are atmost, 3 non-zero elements in each row of $\mathbf{A}_{7}$. In the second stage, $\mathbf{A}_{n-1}$ will be permuted to give a tridiagonal matrix.

The first stage is now illustrated using recursive arguments. Some housekeeping notation is needed to identify pivoted rows. In the real case, the index $t$ was related to the number of transformations carried out $(t-1)$, the rows which have been transformed $(1 \ldots t-1)$ and the next row $(t)$ to be pivoted. The complex case is more complicated; for example, formation of $\mathbf{A}_{\mathbf{4}}$ for the numerical example involves pivoting on row 1,2 and 5 in turn, and the next row to be pivoted is row 3 . It is convenient to define $R_{t}, S_{t}$ and $T_{t}$ to indicate that previous operations have used the first $\left(R_{t}-1\right)$ rows of the first set of rows and the first $\left(S_{t}-1\right)$ rows of the second set of rows as pivots and that $T_{t}$ is the next pivot. $K_{t}$ is used to indicate if the next pivot $\left(T_{t}\right)$ is in the first set of rows $\left(K_{t}=1\right)$ or in the second set of rows $\left(K_{t}=2\right)$. Within the two sets of rows, the rows are transformed in sequence so that $T_{t}=R_{t}$, if $K_{t}=1$, and $T_{t}=n_{1}+S_{t}$, if $K_{t}=2$. Hence, in the numerical example, $R_{4}=2+1 ; S_{4}=5-4+1 ; T_{4}=3 ; K_{4}=1 ; R_{5}=4$. As a total of $(t-1)$ rows of $\mathbf{A}_{1}$ have been pivoted, this equals the sum of rows pivoted in the two sets; i e,$\left(R_{t}-1\right)+\left(S_{t}-1\right)=t-1$. Initially, $t=1, R_{1}=1, S_{1}=1$ and the first row in the first set can be used as the first pivot, and so, $T_{1}=1, K_{1}=1$.

Two slightly different strategies are needed for $K_{t}=1$ and $K_{t}=2$. The derivation of $\mathbf{A}_{t+1}$ is given first when $K_{t}=1$. To simplify notation in this $t^{\text {th }}$ stage, let $r=R_{t}, s=S_{t}, k=K_{t+1}$, and suppose $\mathbf{A}_{t}$ is of the form (A1). As $K_{t}=1$, then the next row to be pivoted is $r$, so $T_{t}=r$; the number of rows in the two sets already used as pivots in $\mathbf{A}_{t+1}$ will be $r$, and $s-1$, so that $R_{t+1}=r+1$ and $S_{t+1}=s$. 
A matrix $\mathbf{A}_{t+1}$ is required, so that $\mathbf{A}_{t+1}=\mathbf{P}_{t} \mathbf{A}_{t} \mathbf{P}_{t}^{\prime}$. Using the same argument as in usual Householder transformations:

$$
\mathbf{P}_{t}=\left[\begin{array}{cc}
\mathbf{I}-\mathbf{u}_{t 1} \mathbf{u}_{t 1}^{\prime} / H_{t} & i \mathbf{u}_{t 1} \mathbf{u}_{t 2}^{\prime} / H_{t} \\
i \mathbf{u}_{t 2} \mathbf{u}_{t 1}^{\prime} / H_{t} & \mathbf{I}-\mathbf{u}_{t 2} \mathbf{u}_{t 2}^{\prime} / H_{t}
\end{array}\right]
$$

with $H_{t}=1 / 2\left(\mathbf{u}_{t 1}^{\prime} \mathbf{u}_{t 1}-\mathbf{u}_{t 2}^{\prime} \mathbf{u}_{t 2}\right)$,

where $\mathbf{u}_{t 1}^{\prime}=\left[0 \ldots 0, b_{r(r+1)}+f_{1}, b_{r(r+2)} \ldots b_{r n_{1}}\right]$

$$
i \mathbf{u}_{t 2}^{\prime}=i\left[0 \ldots 0, c_{r s}+f_{2}, c_{r(s+1)} \ldots c_{r n_{2}}\right]
$$

with terms in (A2) to (A4) expressed as real numbers where possible. A sum of squares $\sigma_{t}$ is needed, given by:

$$
\sigma_{t}=\sum_{m=r+1}^{n_{1}} b_{r m}^{2}-\sum_{m=s}^{n_{2}} c_{r m}^{2}
$$

with $b_{r m}, c_{r m}$ and $d_{r m}$ being elements of the real matrices $\mathbf{B}, \mathbf{C}, \mathbf{D}$, that together form $\mathbf{A}_{t}$ (using (A1)). The elements of $f_{1}$ and $f_{2}$, added to $u_{t 1}$ and $u_{t 2}$, are given by,

$$
\begin{aligned}
& f_{1}=\operatorname{sign}\left(b_{r(r+1)}\right)\left|\sigma_{t}\right|^{1 / 2}, f_{2}=0, k=1, \text { if } \sigma_{t} \geq 0 \\
& f_{2}=\operatorname{sign}\left(c_{r s}\right)\left|\sigma_{t}\right|^{1 / 2}, f_{1}=0, k=2, \text { if } \sigma_{t}<0
\end{aligned}
$$

There are two possibilities in (A6): if $\sigma_{t} \geq 0$, then usual Householder transformation is used, and the next pivot will be the $(r+1)^{\text {th }}$ row in the first set $(k=1)$, and the $(r, r+1)$ element of $\mathbf{A}_{t+1}$ will be non-zero. However, if $\sigma_{t}<0$, then the $\left(n_{1}+s\right)^{\text {th }}$ row is used as a pivot $(k=2)$ in order that $\mathbf{A}_{t+1}$ and $\mathbf{P}_{t}$ have the same form as (A1).

Then $A_{t+1}$ can be calculated using:

$$
\begin{aligned}
& \mathbf{p}_{t 1}=\left(\mathbf{B} \mathbf{u}_{t 1}-\mathbf{C} \mathbf{u}_{t 2}\right) / H_{t} \\
& i \mathbf{p}_{t 2}=i\left(\mathbf{C}^{\prime} \mathbf{u}_{t 1}+\mathbf{D} \mathbf{u}_{t 2}\right) / H_{t} \\
& \mathbf{G}_{t}=\left(\mathbf{u}_{t 1}^{\prime} \mathbf{p}_{t 1}-\mathbf{u}_{t 2}^{\prime} \mathbf{p}_{t 2}\right) 2 / H_{t} \\
& \mathbf{q}_{t 1}=\mathbf{p}_{t 1}-\mathbf{G}_{t} \mathbf{u}_{t 1} \\
& i \mathbf{q}_{t 2}=i\left(\mathbf{p}_{t 2}-\mathbf{G}_{t} \mathbf{u}_{t 2}\right)
\end{aligned}
$$

and $\mathbf{A}_{t+1}=\mathbf{A}_{t}-\left[\begin{array}{cc}\mathbf{u}_{t 1} \mathbf{q}_{t 1}^{\prime} & i \mathbf{u}_{t 1} \mathbf{q}_{t 2} \\ i \mathbf{u}_{t 2} \mathbf{q}_{t 1}^{\prime} & -\mathbf{u}_{t 2} \mathbf{q}_{t 2}\end{array}\right]-\left[\begin{array}{cc}\mathbf{q}_{t 1} \mathbf{u}_{t 1}^{\prime} & i \mathbf{q}_{t 1} \mathbf{u}_{t 2}^{\prime} \\ i \mathbf{q}_{t 2} \mathbf{u}_{t}^{\prime} & -\mathbf{q}_{t 2} \mathbf{u}_{t 2}^{\prime}\end{array}\right]$

For the numerical example with $T_{1}=1$, then from (A5) and (A7), $\sigma_{1}=0.375$; $f_{1}=-0.6124 ; f_{2}=0 ; k=\mathbf{K}_{2}=1$.

So from (A4):

$$
\begin{aligned}
& \mathbf{u}_{11}=\left(\begin{array}{llll}
0.0000 & -2.1124 & -0.7500 & -0.8500
\end{array}\right) \\
& \mathbf{u}_{12}=\left(\begin{array}{llll}
1.5000 & -0.5000 & -0.5000 & -0.5000
\end{array}\right)
\end{aligned}
$$

from (A7)-(A10): 


$$
\begin{aligned}
& \mathbf{p}_{11}=\left(\begin{array}{llll}
1.0000 & -3.2562 & 1.1281 & 1.1281
\end{array}\right) \\
& \mathbf{p}_{12}=\left(\begin{array}{llll}
-0.3431 & -1.2899 & 0.8165 & 0.8165
\end{array}\right) \\
& \mathbf{G}_{1}=2.2699 \\
& \mathbf{q}_{11}=\left(\begin{array}{llll}
1.0000 & 1.5386 & 2.8305 & 2.8305
\end{array}\right) \\
& \text { and } \mathbf{q}_{12}=\left(\begin{array}{llll}
-3.7479 & -0.1550 & 1.9514 & 1.9514
\end{array}\right)
\end{aligned}
$$

Hence, using (A10), $\mathbf{A}_{2}$ has the form of (A1) with:

$$
\begin{aligned}
\mathbf{B} & =\left[\begin{array}{llll}
3.0000 & & & \\
0.6124 & 9.5000 & & \\
0.0000 & 6.3830 & 7.2458 & \\
0.0000 & 6.3830 & 2.2458 & 7.2458
\end{array}\right] \\
\mathbf{C}^{\prime} & =\left[\begin{array}{rrrr}
0.0000 & -10.7247 & -7.5567 & -7.5567 \\
0.0000 & 1.9419 & 0.7990 & 0.7990 \\
0.0000 & 4.3914 & 4.3788 & 2.3788 \\
0.0000 & 4.3914 & 2.3788 & 4.3788
\end{array}\right] \\
\mathbf{D} & =\left[\begin{array}{rrrr}
-12.4436 & & & \\
1.9415 & -1.0405 & & \\
5.1011 & -0.5982 & -3.1514 & \\
5.1011 & -0.5982 & -1.6512 & -3.1514
\end{array}\right]
\end{aligned}
$$

The first column of $\mathbf{A}_{2}$ has only 1 non-zero off-diagonal element; the $(2,1)$ element. $\mathbf{A}_{2}$ has $\mathbf{R}_{2}=2$ and $S_{2}=1$; as only the first row has been pivoted. As $\sigma_{2}<0$, then $K_{2}=1$, and the next row to be pivoted is 2 and $T_{2}=2$. Equations (A5) and (A7) show that $\sigma_{2}=-75.8750 ; f_{1}=0.0000 ; f_{2}=8.7106 ; K_{3}=k=2$.

Formulae are now presented to show how to pivot on a row in the second set of $\mathbf{A}_{t} i e, K_{t}=2$. These formulae are similar to (A2) to (A10), with some changes in sign. In particular:

$$
\mathbf{P}_{t}=\left[\begin{array}{cc}
\mathbf{I}+\mathbf{u}_{t 1} \mathbf{u}_{t 1}^{\prime} / H_{t} & i \mathbf{u}_{t 1} \mathbf{u}_{t 2}^{\prime} / H_{t} \\
i \mathbf{u}_{t 1} \mathbf{u}_{t 2}^{\prime} / H_{t} & \mathbf{I}+\mathbf{u}_{t 2} \mathbf{u}_{t 2}^{\prime} / H_{t}
\end{array}\right]
$$

with $H_{t}=1 / 2\left(-\mathbf{u}_{t 1}^{\prime} \mathbf{u}_{t 1}+\mathbf{u}_{t 2}^{\prime} \mathbf{u}_{t 2}\right)$,

where $i \mathbf{u}_{t 1}=i\left[0 \ldots 0, c_{r s}+f_{3}, c_{(r+1) s} \ldots c_{n_{1}, s}\right]$

$$
\mathbf{u}_{t 2}=\left[0 \ldots 0, d_{s(s+1)}+f_{4}, d_{s(s+2)}, \ldots d_{s n_{2}}\right]
$$

with $\sigma_{t}=\sum_{m=r}^{n_{1}} c_{m s}+\sum_{m=s+1}^{n_{2}} d_{s m}$

and $f_{3}=\operatorname{sign}\left(c_{r s}\right)\left|\sigma_{t}\right|^{1 / 2}, f_{4}=0, K=1$, if $\sigma_{t}<0$

and $f_{4}=\operatorname{sign}\left(d_{s(s+1)}\left|\sigma_{t}\right|^{1 / 2}, f_{3}=0, K=2\right.$, if $\sigma_{t}>0$ 
Then,

$$
\begin{aligned}
& i \mathbf{p}_{t 1}=i\left(\mathbf{B} \mathbf{u}_{t 1}+\mathbf{C} \mathbf{u}_{t 2}\right) / H_{t} \\
& \mathbf{p}_{t 2}=\left(-\mathbf{C}^{\prime} \mathbf{u}_{t 1}+\mathbf{D} \mathbf{u}_{t 2}\right) / H_{t} \\
& \mathbf{G}_{t}=\left(-\mathbf{u}_{t 1}^{\prime} \mathbf{p}_{t 1}+\mathbf{u}_{t 2}^{\prime} \mathbf{p}_{t 2}\right) / 2 H_{t} \\
& i \mathbf{q}_{t 1}=i\left(\mathbf{p}_{t 1}-\mathbf{c}_{t} \mathbf{u}_{t 1}\right) \\
& \mathbf{q}_{t 2}=\left(\mathbf{p}_{t 2}-\mathbf{c}_{t} \mathbf{u}_{t 2}\right)
\end{aligned}
$$

and $\mathbf{A}_{t+1}=\mathbf{A}_{t}-\left[\begin{array}{cc}-\mathbf{u}_{t 1} \mathbf{q}_{t 1}^{\prime} & i \mathbf{u}_{t 1} \mathbf{q}_{t 2}^{\prime} \\ i \mathbf{u}_{t 2} \mathbf{q}_{t 1}^{\prime} & \mathbf{u}_{t 2} \mathbf{q}_{t 2}^{\prime}\end{array}\right]-\left[\begin{array}{cc}-\mathbf{q}_{t 1} \mathbf{u}_{t 1}^{\prime} & i \mathbf{q}_{t 1} \mathbf{u}_{t 2}^{\prime} \\ i \mathbf{q}_{t 2} \mathbf{u}_{t 1}^{\prime} & \mathbf{q}_{t 2} \mathbf{u}_{t 2}^{\prime}\end{array}\right]$

Using (A11) to (A19), matrix $\mathbf{A}_{4}$ can be constructed from:

$$
\begin{aligned}
& \mathbf{B}=\left[\begin{array}{rrrr}
3.0000 & & & \\
0.6124 & 9.500 & & \\
0.0000 & 0.0000 & 0.7969 & \\
0.0000 & 0.0000 & 0.0769 & 5.1259
\end{array}\right] \\
& \mathbf{C}^{\prime}=\left[\begin{array}{rrrr}
0.0000 & 8.7106 & 0.3864 & 0.0000 \\
0.0000 & 0.0000 & -0.1849 & -1.1901 \\
0.0000 & 0.0000 & 0.1036 & -0.4778 \\
0.0000 & 0.0000 & 0.1036 & 2.5232
\end{array}\right] \\
& \mathbf{D}=\left[\begin{array}{rrrr}
-8.3081 & & & \\
0.0000 & -0.6687 & & \\
0.0000 & -0.0341 & -0.5718 & \\
0.0000 & 0.5969 & 0.3770 & -1.6742
\end{array}\right]
\end{aligned}
$$

with $R_{4}=3, S_{4}=2, T_{4}=3, K_{4}=1$.

Using (A12) to (A19), then:

$$
\begin{aligned}
& R_{5}=4, S_{5}=2, T_{5}=6, K_{5}=2 \\
& R_{6}=4, S_{6}=3, T_{6}=4, K_{6}=1 \\
& R_{7}=5, S_{7}=3, T_{7}=7, K_{7}=2
\end{aligned}
$$

$\mathbf{A}_{7}$ is indicated above.

Hence, a matrix has been constructed with, at most, $2(n-1)$ off-diagonal elements. In this example, there are 12 , as the $(6,4)$ and $(4,6)$ elements are zero.

The second stage is to transform $\mathbf{A}_{t-1}$ to tridiagonal form $\mathbf{A}_{t-1}^{*}$. This can be simply achieved by recording the rows and columns in the order that the pivoting was carried out; ie, $T_{t}=(t=1, \ldots, n)$, where $T_{n}$ is the row not included in the set $\left(T_{1}, T_{2}, \ldots, T_{n-1}\right)$, and is either $n_{1}$ or $\left(n_{1}+n_{2}\right)$.

In the numerical example: $T_{1}=1, T_{2}=2, T_{3}=5, T_{4}=3, T_{5}=6, T_{6}=4$, $T_{7}=7$, so $T_{8}=8$ and: 
$\mathbf{A}_{7}^{*}=\left[\begin{array}{ccccccccc}3.0000 & & & & & & & \\ 0.6124 & 9.5000 & & & & & & \\ 0.0000 & 8.7106 i & -8.3081 & & & & & & \\ 0.0000 & 0.0000 & 0.3864 i & 0.7969 & & & & \\ 0.0000 & 0.0000 & 0.0000 & 0.2230 i & -0.4888 & & & \\ 0.0000 & 0.0000 & 0.0000 & 0.0000 & 0.0000 & 3.7419 & & \\ 0.0000 & 0.0000 & 0.0000 & 0.0000 & 0.0000 & 0.1542 i & -0.5228 & \\ 0.0000 & 0.0000 & 0.0000 & 0.0000 & 0.0000 & 0.0000 & 0.2179 & -0.5198\end{array}\right]$

Formation of equations (12) to (15)

If $\left(\mathbf{A}_{n}^{*}+\lambda I\right)$ and $\mathbf{q}^{*}$ are real matrices, say $\mathbf{G}$ and $\mathbf{q}$, then Smith and Graser (1986) suggest writing $\mathbf{G}$ as $\mathbf{U}^{\prime} \mathbf{D} \mathbf{U}$ (where $\mathbf{U}$ is a lower triangular matrix with diagonal elements 1 , and $\mathbf{D}$ is a diagonal matrix) and give an algorithm to find the non-zero elements of $\mathbf{D}$ and $\mathbf{U} ; i e, \mathbf{D}_{j}$ and $\mathbf{U}_{j, k}$.

$$
\begin{aligned}
& D_{1}=G_{11} \\
& U_{j, j-1}=G_{j, j-1} / D_{j} \\
& D_{j}=G_{i j}-G_{j, j-1} U_{j, j-1}
\end{aligned}
$$

and the other elements of $\mathbf{D}$ and $\mathbf{G}$ are zero.

Then $\operatorname{tr}\left(\mathbf{G}^{-1}\right)=\sum w_{j}$ where,

$$
\begin{aligned}
& w_{1}=1 / D_{1} \\
& w_{j}=\left(1+G_{j, j-1} U_{j, j-1} w_{j-1}\right) / D_{j}, \text { when } j>1
\end{aligned}
$$

The elements of $\mathbf{a}^{+}=\mathbf{G}^{-1} q$ can be found by eliminating the elements of $\mathbf{a}^{+}$in turn, and back-substituting to find $a_{j}^{+}$. That is in the first place form:

$$
\begin{aligned}
& a_{1}^{++}=q_{1} \\
& a_{j}^{++}=q_{j}-a_{j-1} U_{j, j-1} \quad(j=2, \ldots, n)
\end{aligned}
$$

Then $a_{n}^{+}=a_{n}^{+} / D_{n}$

$$
\text { and, } a_{j}^{+}=a_{j}^{++} / D_{j}-U_{j+1, j} a_{j+1}^{+} \quad(j=n-2, n-3, \ldots)
$$

Expression (13) can be found from either $a_{j}^{++2} / D_{j}$ or $a_{j}^{+} a_{j}^{++}$.

In our case, $\left(\mathbf{A}_{n}^{*}+\lambda \mathbf{I}\right), q^{*}$ and $\mathbf{a}^{+}$have real and imaginary terms. The indices $K_{j}$ indicate if the elements of $\left(\mathbf{A}_{n}^{*}+\lambda \mathbf{I}\right), \mathbf{q}^{*}$ and $\mathbf{a}^{+}$are real or imaginary. Suppose $\mathbf{G}, \mathbf{q}$ and a contain the real coefficients of $\left(\mathbf{A}_{n}^{*}+\lambda \mathbf{I}\right), \mathbf{q}^{*}$ and $\mathbf{a}^{+}$so that:

$$
\begin{aligned}
& g_{j k}=\left(\mathbf{A}_{n}^{*}+\lambda \mathbf{I}\right)_{j k}, \text { if } K_{j}=K_{k} \\
& i G_{j k}=\left(\mathbf{A}_{n}^{*}+\lambda \mathbf{I}\right)_{j k}, \text { if } K_{j}=K_{k} \\
& q_{j}=q_{j}^{*} \text { and } a_{j}=a_{j}^{*}, \text { if } K_{j}=1 \\
& i q_{j}=q_{j}^{*} \text { and } i a_{j}=a_{j}^{+}, \text {if } K_{j}=2
\end{aligned}
$$


Then Smith and Graser's algorithm can be used again, and in terms of real arithmetic if 3 extra sets of coefficients are defined. There are 4 cases to consider:

(i) $K_{j-1}=1, K_{j}=1$, then $f_{j}=1, f_{j}^{*}=1, f_{j-1}^{* *}=1$

(ii) $K_{j-1}=1, K_{j}=2$, then $f_{j}=-1, f_{j}^{*}=1, f_{j-1}^{* *}=-1$

(iii) $K_{j-1}=2, K_{j}=1$, then $f_{j}=-1, f_{j}^{*}=-1, f_{j-1}^{* *}=1$

(iv) $K_{j-1}=2, K_{j}=2$, then $f_{j}=1, f_{j}^{*}=1, f_{j-1}^{* *}=1$

The real coefficients associated with $\mathbf{U}$ and $\mathbf{D}$ are now given by:

$$
\begin{array}{ll}
D_{1}=G_{11} & \\
U_{j j-1}=G_{j, j-1} / D_{j} & \\
D_{j}=D_{j}-f_{j} G_{j, j-1} U_{j, j-1} \quad(j=2, \ldots, n)
\end{array}
$$

Then $\operatorname{tr}\left(\mathbf{A}_{n}^{*}+\lambda I\right)=\sum w_{j}$

where, $w_{1}=1 / D_{1}$,

and $w_{j}=\left(1+f_{j} G_{j, j-1} U_{j, j-1} w_{j}\right) / D j$

The real coefficients of $\mathbf{a}^{+}, \mathbf{a}$, can be found from

$$
\begin{array}{ll}
a_{1}^{++} & =q_{1} \\
a_{j}^{++} & =q_{j}-f_{j}^{*} a_{j-1}^{++} U_{j, j-1} \quad(j=2, \ldots, n)
\end{array}
$$

Then, $a_{1}^{+}=a_{n}^{++} / D_{n}$,

$$
\text { and } a_{j}=a_{j}^{++} / D_{j}-f_{j}^{* *} U_{j+1, j} a_{j 1}^{+} \quad(j=n-2, n-3, \ldots, 1) \text {, }
$$

and $q^{*}\left(A_{n}+I\right)^{-1} q^{*}=\left(3-2 K_{j}\right) a_{j}^{++} / D_{j}=\left(3-2 K_{j}\right) a_{j}^{+} a_{j}^{+}$.

For instance, for the numerical example:

$\begin{array}{lrrrrrrrr}j= & 1 & 2 & 3 & 4 & 5 & 6 & 7 & 8 \\ K_{j} & 1 & 1 & 2 & 1 & 2 & 1 & 2 & 2 \\ K_{j-1} & & 1 & 1 & 2 & 1 & 2 & 1 & 2 \\ f_{j} & & 1 & -1 & -1 & -1 & -1 & -1 & -1 \\ f_{j}^{*} & & 1 & 1 & -1 & -1 & 1 & 1 & 1 \\ f_{j}^{* *} & 1 & -1 & 1 & 1 & 1 & -1 & 1 & \\ 3-2 K_{j} & & 1 & 1 & -1 & 1 & -1 & -1 & -1\end{array}$

Application of these formulae, with $\mathbf{A}_{7}^{*}$ given in the numerical example, are given in Table $\mathrm{I}$. 
Table I. Application of formula involved in calculating terms in estimating equations.

\begin{tabular}{rrrrrrrrr}
\hline$j$ & $G_{j j}$ & $G_{j+1, j}$ & \multicolumn{1}{c}{$D_{j}$} & $U_{j+1, j}$ & \multicolumn{1}{c}{$q_{j}$} & \multicolumn{1}{c}{$a_{j}^{++}$} & \multicolumn{1}{c}{$a_{j}^{+}$} & $w_{j}$ \\
\hline 1 & 50.0000 & 0.6124 & 5.0000 & 0.1225 & 10.0000 & 10.0000 & 3.1248 & 0.2000 \\
2 & 11.5000 & 8.7106 & 11.4250 & 0.7624 & -21.6272 & -22.8620 & -9.1836 & 0.0888 \\
3 & -6.3081 & 0.3864 & 0.3330 & 1.1604 & -22.0279 & -4.5975 & -9.4208 & 1.2312 \\
4 & 2.7969 & 0.2230 & 3.2453 & 0.0687 & -7.0108 & -12.3455 & -3.7795 & 0.1380 \\
5 & 1.5112 & 0.0000 & 1.5265 & 0.0000 & -0.2994 & 0.5489 & 0.3596 & 0.6537 \\
6 & 5.7419 & 0.1542 & 5.7419 & 0.0269 & -11.1577 & -11.1577 & -1.9134 & 0.1742 \\
7 & 1.4772 & 0.2179 & 1.4813 & 0.1471 & 0.7219 & 1.0215 & 1.1109 & 0.6746 \\
8 & 1.4802 & - & 1.4482 & - & -3.9968 & -4.1471 & -2.8633 & 0.7055 \\
\hline
\end{tabular}

The sum of squares is then 58.1417 , and the trace 3.8360 